\title{
Problem-solving ability and repetition of deliberate self-harm: a multicentre study
}

\author{
CARMEL MCAULIFFE ${ }^{1 *}$, PAUL CORCORAN ${ }^{1}$, HELEN S. KEELEY ${ }^{1}$, \\ ELLA ARENSMAN ${ }^{1}$, UNNI BILLE-BRAHE ${ }^{2}$, DIEGO DE LEO ${ }^{3}$, SANDOR FEKETE ${ }^{4}$, \\ KEITH HAWTON ${ }^{5}$, HEIDI HJELMELAND ${ }^{6}$, MARGARET KELLEHER ${ }^{1}$, \\ AD J.F.M. KERKHOF ${ }^{7}$, JOUKO LÖNNQVIST ${ }^{8}$, KONRAD MICHEL ${ }^{9}$, \\ ELLINOR SALANDER-RENBERG ${ }^{10}$, ARMIN SCHMIDTKE 11 , \\ KEES VAN HEERINGEN ${ }^{12}$ AND DANUTA WASSERMAN ${ }^{13}$ \\ ${ }^{1}$ National Suicide Research Foundation, Cork, Ireland; ${ }^{2}$ Unit for Suicidological Research, Odense, Denmark; \\ ${ }^{3}$ Australian Institute for Suicide Research and Prevention, Griffith University, Brisbane, Australia; \\ ${ }^{4}$ Department of Psychiatry, University of Pecs, Pecs, Hungary; ${ }^{5}$ Centre for Suicide Research, University of \\ Oxford, England; ${ }^{6}$ Department of Social Work and Health Science, The Norwegian University of Science and \\ Technology, Trondheim, Norway; ${ }^{7}$ Department of Clinical Psychology, Vrije Universiteit, Amsterdam, The \\ Netherlands; ${ }^{8}$ Department of Mental Health and Alcohol Research, National Public Health Institute, Helsinki, \\ Finland; ${ }^{9}$ University Psychiatric Services Bern, Outpatients Clinic, Bern, Switzerland; ${ }^{10}$ Department of \\ Clinical Sciences, Division of Psychiatry, Umea University, Sweden; ${ }^{11}$ Clinic and Polyclinic for Psychiatry and \\ Psychotherapy, University of Wurzburg, Germany; ${ }^{12}$ Department of Psychiatry, University of Gent, Belgium; \\ ${ }^{13}$ Department of Public Health Sciences, National and Stockholm County Council's Centre for Suicide \\ Research and Prevention (NASP) at the National Institute for Psychosocial Factors and Health/Karolinska \\ Institute, Stockholm, Sweden
}

\begin{abstract}
Background. While recent studies have found problem-solving impairments in individuals who engage in deliberate self-harm (DSH), few studies have examined repeaters and non-repeaters separately. The aim of the present study was to investigate whether specific types of problem-solving are associated with repeated DSH.
\end{abstract}

Method. As part of the WHO/EURO Multicentre Study on Suicidal Behaviour, 836 medically treated DSH patients (59\% repeaters) from 12 European regions were interviewed using the European Parasuicide Study Interview Schedule (EPSIS II) approximately 1 year after their index episode. The Utrecht Coping List (UCL) assessed habitual responses to problems.

Results. Factor analysis identified five dimensions - Active Handling, Passive-Avoidance, Problem Sharing, Palliative Reactions and Negative Expression. Passive-Avoidance - characterized by a pre-occupation with problems, feeling unable to do anything, worrying about the past and taking a gloomy view of the situation, a greater likelihood of giving in so as to avoid difficult situations, the tendency to resign oneself to the situation, and to try to avoid problems - was the problem-solving dimension most strongly associated with repetition, although this association was attenuated by self-esteem.

Conclusions. The outcomes of the study indicate that treatments for DSH patients with repeated episodes should include problem-solving interventions. The observed passivity and avoidance of problems (coupled with low self-esteem) associated with repetition suggests that intensive therapeutic input and follow-up are required for those with repeated DSH.

\footnotetext{
* Address for correspondence: Carmel McAuliffe, National Suicide Research Foundation, 1 Perrott Avenue, College Road, Cork, Ireland. (Email: carmel.nsrf@iol.ie)
} 


\section{INTRODUCTION}

Repetition of deliberate self-harm (DSH) or parasuicide is common. The WHO/EURO Multicentre Study on Suicidal Behaviour found that $56 \%$ of patients medically treated for DSH had made previous attempts and that $29 \%$ made a further attempt within 12 months of their index act (Kerkhof \& Arensman, 2004). Rates of repetition are increasing (Hawton et al. 1997) and those presenting to hospital with DSH are now more likely to have a history of $\mathrm{DSH}$ and to engage in further acts, than was previously observed (Henriques et al. 2004). Repetition is regarded as an important outcome following an episode of DSH (Owens et al. 1994; Kerkhof \& Arensman, 2004) because it reflects ongoing or recurrent distress (Hawton \& Fagg, 1995; Hawton et al. 1999) and is associated with increased risk of suicide (Zahl \& Hawton, 2004). Repetition is the most commonly used outcome measure in treatment evaluation studies, which may be due to an individual's inability to resolve his or her problems and difficulties. There is evidence to suggest that the acquisition of problem-solving skills may reduce the likelihood of repetition of DSH (Linehan et al. 1987; Hawton et al. 1998) and may also bring about better results than control treatment with regard to depression, hopelessness and problems (Townsend et al. 2001).

While problem-solving impairment has been found to be significantly associated with DSH (Linehan et al. 1987; McLeavey et al. 1987; Rotheram-Borus et al. 1990; Pollock \& Williams, 1998, 2004), few studies have compared problem solving of repeaters with that of non-repeaters. Compared with suicide ideators and first-ever attempters, repeaters have been found to score significantly lower on problemsolving confidence and personal control and significantly higher on approach-avoidance (Rudd et al. 1996). In studies of patients with a diagnosis of borderline personality disorder (BPD), the generation of inappropriate, and to a lesser extent, passive problem solutions [as measured by the Means-Ends Problem Solving scale (MEPS)] were found to be predictive of repetition within 12 months (Kehrer \& Linehan, 1996) while poorer engagement in the coping strategy of comforting cognitions has been independently associated with repetition (Rietdijk et al. 2001).

In one prospective study of hospital-treated DSH patients (Sakinofsky \& Roberts, 1990), greater perceived severity of personal problems was associated with increased risk of repetition within 3 months, although the rate of repetition was similar among those who had problems that resolved and those who did not. Problem score was the first variable to emerge from a stepwise discriminant function analysis that included gender along with 17 other important baseline continuous variables including previous $\mathrm{DSH}$, suicide intent, lethality, depression score, locus of control, powerlessness and other variables. In a prospective 18-month follow-up study of 50 consecutive hospital-treated DSH patients (Dieserud et al. 2003), low self-appraised problem-solving capacity and a low sense of self-efficacy (i.e. a lack of trust in one's own ability to cope with problems) predicted repetition of attempt within 18 months, even when sex, age, previous suicide attempt, suicide intent and lethality were controlled for. Low selfefficacy was the best predictor of repetition. Baseline assessment was carried out an average of 20.4 days following the index attempt, which suggests that self-efficacy is a stable predictor of repetition. The main methodological limitations of these studies is their use of different terminology (attempted suicide, parasuicide, deliberate self-harm) their small sample sizes and in several cases, the use of a single diagnostic group (BPD), which limits the extent to which the study findings can be generalized.

The principal aim of the present study is to compare problem-solving dimension scores of repeaters with non-repeaters from a large sample of patients presenting with DSH, and to investigate whether any of these problemsolving styles is independently associated with repetition of $\mathrm{DSH}$.

\section{METHOD}

\section{Participants}

The participants were 836 medically treated DSH patients from 12 European regions who were followed-up in the Repetition Prediction part of the WHO/EURO Multicentre Study on Suicidal Behaviour. The WHO Working Group of the WHO/EURO Multicentre Study 
on Suicidal Behaviour devised the following definition of parasuicide/attempted suicide:

An act with nonfatal outcome in which an individual deliberately initiates a non-habitual behaviour, that without intervention from others will cause self-harm, or deliberately ingests a substance in excess of the prescribed or generally recognised therapeutic dosage and which is aimed at realising changes that the person desires via the actual or expected physical consequences (Platt et al. 1992).

This definition includes acts that are interrupted before a self-harm injury is sustained, for example a person removed from a bridge before jumping off, but excludes episodes of self-harm by individuals who do not understand the meaning or the outcome of their act, for example, due to a learning disability or severe mental disorder (Bille-Brahe et al. 1994).

The terms 'parasuicide', 'attempted suicide' and 'deliberate self-harm' were used interchangeably by the WHO/EURO Multicentre Study on Suicidal Behaviour. The term 'deliberate self-harm' (DSH) is used in the present paper to reflect the diversity of motives involved in this behaviour, ranging from the 'wish to die' to 'trying to get attention'. However, we acknowledge that the WHO study definition was not intended as a definition of DSH. Across 12 participating regions, a non-consecutive sample of 1598 DSH patients aged 15 years and over who were medically treated for an episode meeting the WHO definition of parasuicide as outlined earlier were interviewed using the European Parasuicide Study Interview Schedule I (EPSIS I). These interviews generally took place within 72 hours of the DSH act. Followup interviews were carried out using EPSIS II with 836 of these individuals $(52 \cdot 3 \%)$ approximately 1 year later. On the basis of the relevant sections of EPSIS I and II, repeaters were identified as patients who had engaged in more than one DSH act. Non-repeaters were those patients whose index act was their only known episode of DSH at the time of follow-up.

\section{Measures}

EPSIS I and II are structured interview schedules that include a number of standardized and non-standardized scales assessing several areas of inquiry, including suicide intent, severity of depressed mood, levels of hopelessness and self-esteem, precipitating problems, and lifetime experiences.

Problem solving was assessed using the 26-item version of the Utrecht Coping List (UCL; Schreurs et al. 1988) at follow-up (EPSIS II). The scale assesses characteristic style of reacting to problems, e.g. ' using a direct approach in order to solve a problem' and also situationspecific coping, e.g. 'showing one's anger with those responsible for the problem'. Each item is positively scored on a four-point Likert response format measuring frequency of reaction ('Seldom or Never', 'Sometimes', 'Often', 'Very Often'). Higher scores indicate greater use of the problem-solving approach. The scale is theoretically based on the assumption that types of coping are not mutually exclusive but operate in various combinations. Schreurs et al. (1988) propose that coping can be categorized into three main types: changing the situation or problem; changing the perception of the situation; or reducing the arousal. The original factor structure of the UCL is composed of the following seven problem-solving dimensions, providing separate scores on each dimension rather than an overall composite score.

Active Handling (6 items). This is characterized by an active approach to problem solving in which steps are taken to solve the problem itself, i.e. changing the situation. The problem is approached directly, thought about, and several solutions are considered and planned, e.g. 'Making several alternative plans for handling a problem'.

Palliative Reactions (4 items). This involves efforts at changing the feelings elicited by the problem, i.e. changing the arousal, which include a number of avoidance strategies such as distraction or time out, e.g. 'Directing one's thoughts towards other matters'.

Avoidance/Wait (3 items). Avoiding or resigning oneself to the problem, i.e. not changing the problem itself, e.g. 'Trying to avoid difficult situations as much as possible'.

Seek Social Support (3 items). Seeking comfort, support and sympathy from others, i.e. changing one's perception and arousal, e.g. 'Sharing one's worries with someone'. 
Passive Reactions (4 items). Feeling helpless, pessimistic and overwhelmed by the problem, i.e. not changing the perception or arousal, e.g. 'Being totally pre-occupied with the problems'.

Expression of Emotions (4 items). To express one's feelings about the problem (including anger and annoyance) to others, i.e. to change one's arousal, e.g. 'Showing one's anger with those responsible for the problem'.

Comforting Cognitions (2 items). To engage in self-comforting and reassuring thoughts, i.e. changing one's perception of the problem and changing one's arousal, e.g. 'Telling oneself that other people also have their problems from time to time'.

The internal consistency of the UCL has been examined among different norm groups, generally revealing relatively similar values of Cronbach's $\alpha$ (Schreurs et al. 1993). Based on a large sample of mainly male employees of the Dutch Railway Company $(n=1200,93 \%$ males), the alphas appeared to be ranging from sufficient $(0.64)$ for the subscale Expression of Emotions to good (0.82) for the subscale Active Handling. A study among 164 medical students with an equal representation of males and females revealed alphas ranging from sufficient $(0.63)$ for the subscales Palliative Reactions and Comforting Cognitions to good (0.88) for the subscale Seek Social Support.

With regard to the construct validity of the UCL, evidence has been found for two underlying dimensions: (1) a reactive and defensive coping style, represented by the subscales Avoidance, Passive Reactions and Expression of Emotions, explaining 35\% of the variance and (2) an active and assertive coping style, represented by the subscales Active Handling, Comforting Cognitions and Seek Social Support explaining $19 \%$ of the variance. The subscale Palliative Reactions appeared to be represented on both dimensions. With regard to the question as to whether the UCL coping styles should be interpreted as 'trait' or 'state' measures, Sanderman \& Ormel (1992) found that the subscales are relatively stable and, therefore, should be positioned between trait and state measures.

Suicide intent was assessed using the Suicide Intent Scale at EPSIS I (SIS; Beck et al. 1974a).
It consists of 15 items, which assess the severity of the wish to die associated with a recent episode of self-harm. Each item is scored 0-2 giving a total score range of $0-30$, with higher scores indicating a greater suicide intention.

Levels of depressive symptomatology were assessed at EPSIS I using the Beck Depression Inventory (BDI; Beck et al. 1961), a 21-item scale that assesses the severity of depression in psychiatrically diagnosed adolescents and adults. Respondents are asked to indicate on a series of statement groupings, which statement best describes how they have been feeling over the previous several days, e.g. 'I feel disappointed in myself'. Responses are scored on a 0-3 Likert scale and summed to provide a global depression score.

Scores obtained on the Beck Hopelessness Scale (BHS; Beck et al. 1974b) administered at the EPSIS I interview were also examined. This 20-item questionnaire assesses negative expectations and pessimism about one's future (e.g. 'I look forward to the future with hope and enthusiasm') and is scored on a true-false style response format. Half of the items are keyed in the opposite direction to minimize response bias. Each item is scored 0 or 1 and summed to provide a total score ranging from $0-20$, with higher scores indicating greater hopelessness.

Self-esteem was assessed in both EPSIS I and II interviews using the Rosenberg Self-Esteem Scale (RSE; Rosenberg, 1965) a 10-item selfreport scale that assesses respondents' current level of self-esteem and global self-worth (e.g. 'At times I think I am no good at all'). Each item is scored on a four-point Likert response format, measuring level of agreement (ranging from 'strongly agree' to 'strongly disagree') Total scores range from 10 to 40 , with higher scores indicating higher self-esteem. The RSE has been found to have high internal consistency $(\alpha=0 \cdot 89)$ in a study by Dori \& Overholser (1999) and adequate test-retest reliability (Fleming \& Courtenay, 1984).

The General Health Questionnaire (GHQ-30; Goldberg et al. 1976) was administered as part of the EPSIS II assessment schedule. The GHQ was designed as a self-report screening test for the purpose of detecting psychiatric disorders in respondents (e.g. 'Have you recently been feeling unhappy and depressed?'). The shortened 30item GHQ is one of the most commonly used 
and the most widely validated versions. Responses are scored $0,0,1,1$ in ascending order, with higher scores indicating greater disorder. The internal consistency of the GHQ-30 has been examined among a number of different norm groups, all of which appear to reveal similar values of Cronbach's $\alpha$. The alphas appear to be good, ranging from 0.84 in a sample of students in Hong Kong $(n=72)$ (Chan, 1985), to 0.90 in a community sample (Goodchild \& Duncan-Jones, 1985), to 0.93 in a sample of English schoolchildren $(n=129$; Keyes, 1984). In terms of validity, Goldberg \& Williams (1988) report a median sensitivity score (based on 29 studies of the GHQ-30 against the Diagnostic Interview Schedule) of $81 \%$. The median specificity of the GHQ-30 in these same studies was $80 \%$.

Problem drinking was established on the basis of responses to two measures - the CAGE questionnaire (Ewing, 1984) administered at EPSIS I, and the question: 'Do you consider alcohol to be a problem for you at the present time?' as asked at EPSIS II. The CAGE is a brief four-item questionnaire used to detect alcoholism in adolescents and adults, e.g. 'In the past year, have you ever felt guilty about your drinking behaviour?' Those responding 'yes' to two or more items were categorized as problem drinkers. Sensitivity rates of $93 \%$ and $93.8 \%$ respectively and specificity rates of $76 \%$ and $85.5 \%$ respectively, have been reported for the CAGE in the identification of problem drinkers in a sample of psychiatric patients (Bernadt et al. 1982) and in a sample of general medical inpatients (Castells \& Furlanetto, 2005). Those who indicated in response to the question: 'Do you consider alcohol to be a problem for you at the present time?' that alcohol was either a minor or a major problem for them were categorized as problem drinkers.

\section{Statistical analysis}

The EPSIS II sample was compared with those who only completed EPSIS I with respect to a range of variables using the $\chi^{2}$ test and Student's $t$ test as appropriate. Factor analysis using principal components was used to examine the structure of the responses. Varimax rotation was used to aid the interpretation of the derived factors. Comparison was made with the original seven-factor structure.
One-way ANOVA was used to examine the effects of repeater status, gender and their interaction on the scores on the five problemsolving dimensions derived from the factor analysis. Separate logistic regression analysis was carried out to assess the association between each of the problem-solving dimensions and repeater status. All five problem-solving dimensions were then entered into a single multivariate model with sex, age, self-esteem and general health scores and problem drinking, to assess which variables were independently associated with repetition. In the logistic regression analyses, problem-solving, self-esteem and general health scores were categorized as low, medium and high based on the lower, middle and upper tertiles. Because the data were derived from 12 regions, we investigated whether the results of the logistic regression changed when the clustered nature of the data was taken into account. A two-level multilevel logistic regression model with centre defined as the higher level showed almost identical results, indicating that there was no effect of the clustering of the data. Therefore, only the results from the standard logistic regression analyses are presented.

\section{RESULTS}

\section{Sample}

Table 1 describes the EPSIS II sample in comparison with those who completed the EPSIS I interview only, with respect to 11 variables that relate to their demographic characteristics, index act and psychological symptoms assessed at EPSIS I interview. Using Bonferroni's adjustment (significance level $=0 \cdot 05 / 11=0 \cdot 0045$ ), the EPSIS II sample was significantly different to the EPSIS I sample only in relation to three variables: gender, education and problem drinking. Those who were not successfully followed-up were more likely to be men and to have a low level of education and to score within the range of problem drinking on the CAGE questionnaire.

\section{Factor analysis of responses to the $\mathrm{UCL}$}

The factor analysis of the 26-item UCL yielded five factors as opposed to the seven factors originally derived by the developers of the scale (Table 2). The five factors accounted for $53.0 \%$ of the total variance. The five factors Active 
Table 1. Comparison of EPSIS II sample with EPSIS I only sample based on characteristics assessed at EPSIS I

\begin{tabular}{|c|c|c|c|c|c|}
\hline Characteristic & $\begin{array}{l}\text { EPSIS II } \\
(n=836)\end{array}$ & $\begin{array}{l}\text { EPSIS I only } \\
\quad(n=762)\end{array}$ & $\chi^{2} /$ Student's $t$ & df & $p$ value \\
\hline \multicolumn{6}{|l|}{ Gender } \\
\hline Male & $303(36 \%)$ & $330(43 \%)$ & $8 \cdot 205$ & 1 & $0 \cdot 004$ \\
\hline Female & $532(64 \%)$ & $432(57 \%)$ & & & \\
\hline Age (mean) & 36 years & 35 years & $t=1 \cdot 554$ & 1585 & $0 \cdot 120$ \\
\hline \multicolumn{6}{|l|}{ Marital status } \\
\hline Single & $389(47 \%)$ & $367(48 \%)$ & $3 \cdot 191$ & 4 & $0 \cdot 526$ \\
\hline Married & $222(27 \%)$ & $184(24 \%)$ & & & \\
\hline \multicolumn{6}{|l|}{ Education level } \\
\hline Low & $338(41 \%)$ & $366(49 \%)$ & $10 \cdot 930$ & 2 & $0 \cdot 004$ \\
\hline Moderate & $399(48 \%)$ & $327(43 \%)$ & & & \\
\hline High & $93(11 \%)$ & $62(8 \%)$ & & & \\
\hline Previous deliberate self-harm at index act & $478(58 \%)$ & $417(57 \%)$ & $0 \cdot 015$ & 1 & $0 \cdot 902$ \\
\hline Suicide intent at index act (mean SIS score) & $13 \cdot 31$ & $13 \cdot 67$ & $t=0.974$ & 1312 & $0 \cdot 330$ \\
\hline \multicolumn{6}{|l|}{ Method of index act } \\
\hline Self-poisoning only & $694(83 \%)$ & $602(80 \%)$ & $4 \cdot 230$ & 2 & $0 \cdot 121$ \\
\hline Self-injury & $88(12 \%)$ & $92(11 \%)$ & & & \\
\hline Both & $46(6 \%)$ & $60(8 \%)$ & & & \\
\hline Problem drinking (CAGE score > 1) & $249(32 \%)$ & $247(37 \%)$ & $4 \cdot 604$ & 1 & $0 \cdot 032$ \\
\hline Depression (mean BDI score) & $24 \cdot 4$ & $24 \cdot 2$ & $t=0 \cdot 269$ & 1327 & $0 \cdot 788$ \\
\hline Hopelessness (mean BHS score) & $10 \cdot 5$ & $11 \cdot 0$ & $t=1.508$ & 1078 & $0 \cdot 132$ \\
\hline Self-esteem (mean RSE score) & $26 \cdot 33$ & $26 \cdot 25$ & $t=0 \cdot 286$ & 1435 & $0 \cdot 775$ \\
\hline
\end{tabular}

EPSIS, European Parasuicide Study Interview Schedule; SIS, Suicide Intent Scale; BDI, Beck Depression Inventory; BHS, Beck Hopelessness Scale; RSE, Rosenberg Self-Esteem Scale.

Using Bonferroni's adjustment, the significance level $=0 \cdot 05 / 11=0 \cdot 0045$.

Handling, Passive-Avoidance, Problem Sharing, Palliative Reactions, and Negative Expression explained $20 \cdot 2 \%, 12 \cdot 1 \%, 8.9 \%, 6.7 \%$ and $5.2 \%$ of the variance respectively, all with eigenvalues $>1$. Based on the Varimax rotation, the original items that loaded on two separate factors (Passive Reactions and Avoidance/Wait) now loaded on one factor that was interpreted as Passive-Avoidance. With the exception of one item, the same items loaded on the Active Handling and Palliative Reactions factors as in the original analysis. The original Expression of Emotions factor was reduced from four to two items ('Showing one's anger with those responsible for the problem' and 'Showing one's annoyance') and was named Negative Expression. The additional items: ('Showing one's feelings' and 'Showing that there are things that are bothering you') now loaded on the Seeking Social Support factor. This factor was named Problem Sharing.

\section{Comparisons of the levels of the five problem-solving dimensions}

The mean scores on four of the five problemsolving dimensions were significantly different when compared by repeater status and gender (Tables 3 and 4). On average, repeaters scored higher on the Passive-Avoidance factor and lower on the Active Handling factor. Repeaters also scored higher on the Negative Expression factor. However, there was a significant interaction between repeater status and gender in relation to this factor (Table 4), whereby repeaters had higher levels of Negative Expression compared with non-repeaters in men only (mean $=3 \cdot 1$ v. $2 \cdot 7, t=3 \cdot 30, \mathrm{df}=250, p<0 \cdot 01)$. Overall, men scored lower on the Problem Sharing factor. The significant interaction effect indicated that this difference was more pronounced among non-repeaters (mean $=7 \cdot 3$ males $v .8 .8$ females, $t=5.04, \mathrm{df}=260, p<0.001)$ than it was among repeaters (mean $=7.5$ males $v$. $8 \cdot 1$ females, $t=2 \cdot 48, \quad \mathrm{df}=383, p<0.05)$. However, while these differences were statistically significant, their magnitude, as summarized by the $\eta^{2}$ statistic, was relatively small.

\section{Relationship between the five problem-solving dimensions and repeater status}

All five problem-solving dimensions together with gender, age, self-esteem scores (Cronbach's 
Table 2. Factor structure of the Utrecht Coping List (UCL)

\begin{tabular}{|c|c|c|c|}
\hline Factor & $\begin{array}{l}\text { No. of } \\
\text { items }\end{array}$ & Items & $\begin{array}{l}\text { Factor } \\
\text { loadings }\end{array}$ \\
\hline \multirow{7}{*}{$\begin{array}{l}\text { Active Handling } \\
(\alpha=0 \cdot 80)\end{array}$} & \multirow[t]{7}{*}{7} & Finding out all about the problem & $0 \cdot 74$ \\
\hline & & Making several alternative plans for handling a problem & $0 \cdot 69$ \\
\hline & & Considering different solutions to the problem & 0.69 \\
\hline & & Making a direct intervention when problems occur & $0 \cdot 65$ \\
\hline & & Using a direct approach in order to solve the problem & $0 \cdot 63$ \\
\hline & & Considering problems as a challenge & $0 \cdot 63$ \\
\hline & & Realising every cloud has a silver lining & $0 \cdot 42$ \\
\hline \multirow{7}{*}{$\begin{array}{l}\text { Passive-Avoidance } \\
(\alpha=0.79)\end{array}$} & \multirow[t]{7}{*}{7} & Being totally pre-occupied with the problems & $0 \cdot 74$ \\
\hline & & Feeling unable to do anything & $0 \cdot 72$ \\
\hline & & Worrying about the past & $0 \cdot 71$ \\
\hline & & Taking a gloomy view of the situation & $0 \cdot 70$ \\
\hline & & Giving in, in order to avoid difficult situations & $0 \cdot 59$ \\
\hline & & Resigning oneself to the situation & $0 \cdot 55$ \\
\hline & & Trying to avoid difficult situations as much as possible & $0 \cdot 45$ \\
\hline \multirow{5}{*}{$\begin{array}{l}\text { Problem Sharing } \\
(\alpha=0.82)\end{array}$} & \multirow[t]{5}{*}{5} & Sharing one's worries with someone & $0 \cdot 76$ \\
\hline & & Asking someone to help & $0 \cdot 75$ \\
\hline & & Seeking sympathy and comfort from somebody & $0 \cdot 75$ \\
\hline & & Showing there are things which are bothering you & $0 \cdot 74$ \\
\hline & & Showing one's feelings & $0 \cdot 70$ \\
\hline \multirow{5}{*}{$\begin{array}{l}\text { Palliative Reactions } \\
(\alpha=0.66)\end{array}$} & \multirow[t]{5}{*}{5} & Looking for distraction & $0 \cdot 73$ \\
\hline & & Directing one's thoughts towards other matters & $0 \cdot 68$ \\
\hline & & Trying to dispel one's worries temporarily by taking a break & $0 \cdot 66$ \\
\hline & & Trying to make oneself feel better one way or the other & $0 \cdot 63$ \\
\hline & & Telling oneself that other people also have their problems from time to time & $0 \cdot 33$ \\
\hline \multirow{2}{*}{$\begin{array}{l}\text { Negative Expression } \\
(\alpha=0.63)\end{array}$} & \multirow[t]{2}{*}{2} & Showing one's anger with those responsible for the problem & $0 \cdot 69$ \\
\hline & & Showing one's annoyance & $0 \cdot 70$ \\
\hline
\end{tabular}

Table 3. Comparison of mean (standard deviation) UCL dimension scores by repeater status and gender

\begin{tabular}{|c|c|c|c|c|c|c|c|c|}
\hline \multirow[b]{2}{*}{ Dimension } & \multicolumn{2}{|c|}{ Non-repeater } & \multicolumn{2}{|c|}{ Repeater } & \multicolumn{2}{|c|}{ Male } & \multicolumn{2}{|c|}{ Female } \\
\hline & Mean & (s.D.) & Mean & (S.D.) & Mean & (s.D.) & Mean & (S.D.) \\
\hline Active Handling & $10 \cdot 7$ & $(2 \cdot 5)$ & $9 \cdot 9$ & $(2 \cdot 8)$ & $10 \cdot 3$ & $(2 \cdot 7)$ & $10 \cdot 0$ & $(2 \cdot 8)$ \\
\hline Passive-Avoidance & $9 \cdot 3$ & $(2 \cdot 6)$ & $11 \cdot 0$ & $(3 \cdot 0)$ & $10 \cdot 4$ & $(3 \cdot 1)$ & $10 \cdot 5$ & $(2 \cdot 9)$ \\
\hline Problem Sharing & $8 \cdot 2$ & $(2 \cdot 5)$ & $7 \cdot 9$ & $(2 \cdot 7)$ & $7 \cdot 4$ & $(2 \cdot 5)$ & $8 \cdot 4$ & $(2 \cdot 7)$ \\
\hline Palliative Reactions & $7 \cdot 1$ & $(1 \cdot 8)$ & $7 \cdot 0$ & $(1 \cdot 8)$ & $7 \cdot 1$ & $(1 \cdot 8)$ & $7 \cdot 0$ & $(1 \cdot 8)$ \\
\hline Negative Expression & $2 \cdot 9$ & $(1 \cdot 0)$ & $3 \cdot 0$ & $(1 \cdot 1)$ & $2 \cdot 9$ & $(1 \cdot 1)$ & $2 \cdot 9$ & $(1 \cdot 0)$ \\
\hline
\end{tabular}

UCL, Utrecht Coping List.

$\alpha=0.90$ ), general health scores (Cronbach's $\alpha=0.97)$ and problem drinking were entered as predictor variables in a multivariate logistic regression analysis with repetition (more than one lifetime DSH act) as the dependent variable. Passive-Avoidance was significantly associated with repetition when considered alongside gender and age (Table 5). High scores on Active Handling were significantly (but more weakly) associated with reduced risk of repetition. There was evidence that the association between Passive-Avoidance and repetition was not independent of self-esteem, as the odds ratio associated with being a repeater for those with high Passive-Avoidance was reduced from 3.61 to 1.72 with adjustment for the effect of selfesteem.

When the repeaters were further categorized so as to identify those who repeated during the follow-up period, of the 785 participants for whom repetition during follow-up could be established, 232 (29.6\%) did repeat. Re-analysis of the data to test whether problem-solving ability at EPSIS II retrospectively predicted repetition of DSH over the ' 12 months' followup period led to similar but weaker associations 
Table 4. ANOVA results comparing the effects of repeater status, gender and their interaction on the Utrecht Coping List (UCL) dimension scores

\begin{tabular}{llrccc}
\hline \hline Dimension & \multicolumn{1}{c}{ Effect } & \multicolumn{1}{c}{$F$} & df & $p$ & $\eta^{2}$ \\
\hline Active & Repeater & $9 \cdot 068$ & 1,749 & $0 \cdot 003$ & $0 \cdot 012$ \\
Handling & Gender & $0 \cdot 629$ & 1,749 & $0 \cdot 428$ & $0 \cdot 001$ \\
& Repeater *Gender & $1 \cdot 822$ & 1,749 & $0 \cdot 177$ & $0 \cdot 002$ \\
Passive- & Repeater & $56 \cdot 410$ & 1,756 & $0 \cdot 000$ & $0 \cdot 069$ \\
Avoidance & Gender & $0 \cdot 542$ & 1,756 & $0 \cdot 462$ & $0 \cdot 001$ \\
& Repeater *Gender & $0 \cdot 097$ & 1,756 & $0 \cdot 755$ & $0 \cdot 000$ \\
Problem & Repeater & $1 \cdot 422$ & 1,750 & $0 \cdot 233$ & $0 \cdot 002$ \\
Sharing & Gender & $28 \cdot 013$ & 1,750 & $0 \cdot 000$ & $0 \cdot 036$ \\
& Repeater *Gender & $5 \cdot 310$ & 1,750 & $0 \cdot 021$ & $0 \cdot 007$ \\
Palliative & Repeater & $0 \cdot 185$ & 1,750 & $0 \cdot 667$ & $0 \cdot 000$ \\
Reactions & Gender & $0 \cdot 432$ & 1,750 & $0 \cdot 511$ & $0 \cdot 001$ \\
& Repeater *Gender & $1 \cdot 490$ & 1,750 & $0 \cdot 223$ & $0 \cdot 002$ \\
Negative & Repeater & $5 \cdot 812$ & 1,762 & $0 \cdot 016$ & $0 \cdot 008$ \\
Expression & Gender & $0 \cdot 648$ & 1,762 & $0 \cdot 421$ & $0 \cdot 001$ \\
& Repeater *Gender & $6 \cdot 515$ & 1,762 & $0 \cdot 011$ & $0 \cdot 008$ \\
\hline
\end{tabular}

Repeater $*$ Gender represents the effect of the interaction of repeater status and gender on the dimension score.

$\eta^{2}$ indicates the proportion of the variation in the UCL dimension scores that is due to the effect of the independent variables.

between the problem-solving dimensions and risk of repetition.

\section{DISCUSSION}

As a specific coping response to problems, a combination of greater passivity and avoidance-described in this study as PassiveAvoidance - is independently associated with increased risk of repetition of DSH, when considered alongside gender and age group. This coping style is characterized by a preoccupation with problems, worrying about the past, feeling unable to do anything and taking a gloomy view of the situation. In addition, this response to problems involves a greater likelihood of giving in, so as to avoid difficult situations, the tendency to resign oneself to the situation, and to try to avoid problems. The association was no longer significant when the remaining four problem-solving dimensions, together with general health, problem drinking and self-esteem were included in the regression.

Particularly after the inclusion of self-esteem, the association between repetition of DSH and high Passive Avoidance was weakened. Higher self-esteem may protect against repetition of DSH in those who score high on PassiveAvoidance. As an individual disposition, selfesteem is believed to moderate the relationship
Table 5. Results of logistic regression analyses to assess the association between the five problemsolving dimensions and repetition

\begin{tabular}{|c|c|c|c|c|}
\hline Dimension/variable & $\mathrm{OR}^{\mathrm{a}}$ & $95 \% \mathrm{CI}$ & $\mathrm{OR}^{\mathrm{b}}$ & $95 \% \mathrm{CI}$ \\
\hline \multicolumn{5}{|l|}{ Active Handling } \\
\hline Low (ref. group) & $1 \cdot 00$ & - & $1 \cdot 00$ & - \\
\hline Medium & $0 \cdot 71$ & $0 \cdot 49-1 \cdot 05$ & 0.93 & $0.57-1 \cdot 52$ \\
\hline High & $0 \cdot 46^{* * *}$ & $0 \cdot 32-0 \cdot 67$ & $0 \cdot 96$ & $0 \cdot 57-1.63$ \\
\hline \multicolumn{5}{|l|}{ Passive-Avoidance } \\
\hline Low (ref. group) & $1 \cdot 00$ & - & $1 \cdot 00$ & - \\
\hline Medium & $2 \cdot 08 * * *$ & $1 \cdot 44-3 \cdot 00$ & 1.44 & $0 \cdot 88-2 \cdot 33$ \\
\hline High & $3 \cdot 61 * * *$ & $2 \cdot 43-5 \cdot 35$ & $1 \cdot 72$ & $0 \cdot 94-3 \cdot 13$ \\
\hline \multicolumn{5}{|l|}{ Problem Sharing } \\
\hline Low (ref. group) & $1 \cdot 00$ & - & $1 \cdot 00$ & - \\
\hline Medium & $0 \cdot 88$ & $0 \cdot 60-1 \cdot 28$ & 1.04 & $0.64-1.69$ \\
\hline High & $0 \cdot 71$ & $0 \cdot 48-1 \cdot 03$ & $0 \cdot 85$ & $0 \cdot 51-1 \cdot 39$ \\
\hline \multicolumn{5}{|l|}{ Palliative Reactions } \\
\hline Low (ref. group) & $1 \cdot 00$ & - & 1.00 & - \\
\hline Medium & $0 \cdot 73$ & $0 \cdot 50-1 \cdot 05$ & $0 \cdot 88$ & $0 \cdot 56-1 \cdot 40$ \\
\hline High & 0.92 & $0 \cdot 63-1 \cdot 35$ & $0 \cdot 91$ & $0 \cdot 56-1 \cdot 49$ \\
\hline \multicolumn{5}{|l|}{ Negative Expression } \\
\hline Low (ref. group) & $1 \cdot 00$ & - & $1 \cdot 00$ & - \\
\hline Medium & $1 \cdot 18$ & $0 \cdot 80-1 \cdot 74$ & $1 \cdot 17$ & $0 \cdot 74-1 \cdot 86$ \\
\hline High & $1 \cdot 40$ & $0 \cdot 95-2 \cdot 06$ & $1 \cdot 31$ & $0 \cdot 81-2 \cdot 13$ \\
\hline \multicolumn{5}{|l|}{ General Health } \\
\hline Low ( $0-20$ ref. group) & - & - & $1 \cdot 00$ & - \\
\hline Medium (21-38) & - & - & $0 \cdot 83$ & $0 \cdot 52-1 \cdot 33$ \\
\hline High (39-90) & - & - & $1 \cdot 18$ & $0 \cdot 65-2 \cdot 13$ \\
\hline \multicolumn{5}{|l|}{ Self-esteem } \\
\hline High (30-40 ref. group) & - & - & $1 \cdot 00$ & - \\
\hline Medium (25-29) & - & - & $1 \cdot 50$ & $0 \cdot 93-2 \cdot 43$ \\
\hline Low $(10-24)$ & - & - & $2 \cdot 28 * *$ & $1 \cdot 22-4 \cdot 27$ \\
\hline \multicolumn{5}{|l|}{ Problem drinking } \\
\hline No (ref. group) & - & - & $1 \cdot 00$ & - \\
\hline Minor problem & - & - & $1 \cdot 38$ & $0 \cdot 78-2 \cdot 43$ \\
\hline Major problem & - & - & $2 \cdot 86 * *$ & $1 \cdot 51-5 \cdot 43$ \\
\hline \multicolumn{5}{|l|}{ Gender } \\
\hline Male (ref. group) & - & - & $1 \cdot 00$ & - \\
\hline Female & - & - & $1 \cdot 06$ & $0 \cdot 71-1 \cdot 59$ \\
\hline \multicolumn{5}{|l|}{ Age (years) } \\
\hline 15-24 (ref.group) & - & - & $1 \cdot 00$ & - \\
\hline $25-34$ & - & - & $1 \cdot 27$ & $0 \cdot 74-2 \cdot 17$ \\
\hline $35-44$ & - & - & $1 \cdot 71$ & $0.98-2.97$ \\
\hline $45-54$ & - & - & $1 \cdot 16$ & $0.68-1.97$ \\
\hline
\end{tabular}

a Odds ratios derived from five separate logistic regression models, each including a problem-solving dimension, gender and age group.

b Odds ratios derived from the multivariate logistic regression model including all five problem-solving dimensions, the general health, self-esteem and problem drinking variables and gender and age group.

*** $p<0.001,{ }^{* *} p<0 \cdot 01,{ }^{*} p<0 \cdot 05$.

between life events and suicidal behaviour. Low self-esteem may increase the likelihood that an individual makes negative appraisals during stressful experiences (Sandin et al. 1998). In an earlier study of hospitalized DSH patients (Petrie et al. 1988) baseline measures of 
self-esteem were found to be the best predictor of a repeated act at 6 months compared with depression, hopelessness, cognitive rigidity, assertiveness and social anxiety. Petrie et al. (1988) proposed that high self-esteem might operate by encouraging a more positive attitude among DSH patients in their ability to deal with problems, acting as a longer term protective factor against repeated DSH. Low self-esteem has also been associated with future DSH among adolescents even when gender and depression are controlled for (Lewinsohn et al. 1994).

Repeaters scored significantly lower than non-repeaters on the Active Handling dimension (tackling problems positively and proactively), and high scores on this dimension were significantly associated with reduced risk of repetition, when considered alongside age and gender. However the association was weaker than that of Passive-Avoidance. Repeaters were also significantly more inclined to engage in negative emotional expression (showing one's anger or annoyance) but this was confined to the males and was not independent of the effects of the other variables. Males were significantly less inclined to share problems although this gender difference was particularly evident among non-repeaters.

Two major aims of the WHO/EURO Repetition-Prediction study were to identify personal and social characteristics predictive of repeated suicidal behaviour; and to evaluate existing scales designed to predict suicidal behaviour. However, unlike many of the other scales included in the EPSIS II interview schedule, the UCL was not used as a baseline assessment (in EPSIS I) but as an outcome measure at follow-up (Kerkhof et al. 1993; Kerkhof, personal communication, 1999). Associations could only be examined between the predictor variables (problem-solving styles, self-esteem, general health, problem drinking) and repetition retrospectively. When the associations were also examined for prospective repeaters, i.e. those who repeated between EPSIS I and II, they were found to be similar but weaker in magnitude. Retrospective studies have already been criticized for their limited utility in the prediction of repetition (Hjelmeland, 1996). Nevertheless, the UCL dimensions are useful in deriving a coping profile of DSH patients and in identifying a distinct coping style associated with repetition, which should now be investigated further in a prospective study.

The attrition rate in this study is similar to rates obtained in previous follow-up studies with this difficult-to-engage population (Arensman \& Kerkhof, 1996; Hawton et al. 1998; Amsel \& Mann, 2001). The most frequently reported reasons for attrition were patients relocating, refusing follow-up in the EPSIS I interview or refusing follow-up when contacted to complete the EPSIS II interview. Although those who were not successfully followed up were more likely to be men, to have a low level of education, and to have a drink problem, the sample interviewed at follow-up were similar to the original sample on variables relating to psychological symptoms (including depression and hopelessness), self-esteem, clinical history (previous DSH), characteristics of the index act (including suicide intent and method used) and demographic characteristics (including age and marital status). We were unable to determine whether EPSIS I interviewees were representative of the population of DSH patients who presented to hospital in the participating regions. A previous comparison based on nine regions found that women and those who had taken an overdose were overrepresented in those interviewed while they were broadly similar in relation to age and previous attempts (Bille-Brahe et al. 1996). It is also conceivable that a passive avoidant approach to problems is influenced by mood, but it was not possible to control for levels of depression or hopelessness in the present study as they were only assessed at EPSIS I interview. An earlier study of problem solving among DSH patients found that while levels of depression, hopelessness and suicide ideation reduced over a 6-week interval, their tendency to engage in passive problem solving stayed the same (Pollock \& Williams, 2004). The effect of mood on Passive-Avoidance in DSH patients with repeated episodes needs to be investigated further.

The findings point to a habitual coping style among DSH repeaters characterized by a combination of passive and or avoidant reactions to problems, that may contribute to a process of sensitization, in which the association between negative life events and episodes of DSH is weakened with an increasing number of episodes (Joiner \& Rudd, 2000). Williams (1997) 
observed that suicidal people stop trying to solve problems because they tend to overgeneralize from a problem that cannot be solved to situations in which things can be done. Passivity and avoidance in relation to problems are likely to be maintained if repeaters feel hopeless when faced with problems (Sakinofsky et al. 1990; Milnes et al. 2002).

These findings may go some way towards informing the content and format of treatment programmes designed to reduce repetition of $\mathrm{DSH}$, given that the optimal treatment approaches are still uncertain, despite promising findings reported from randomized controlled trials (Hawton et al. 1998). The observed passivity and avoidance of problems associated with repetition of DSH in this study indicates the need for intensive therapeutic input and followup, as low self-esteem may hinder clients in coping with problems. In terms of treatment format, the active involvement of a therapist is favourable over self-help-only approaches. The failure of a recent trial (using a cognitive therapeutic approach) to reduce repetition (Tyrer et al. 2003) in which over one-third of the active treatment sample received a treatment manual alone without any treatment sessions, suggests that reliance purely on a self-help approach among DSH repeaters is ineffective in reducing repetition (Arensman et al. 2004). Interventions to reduce repetition are likely to be more effective if based on direct coaching by the therapist in an active and positive approach to problems through appropriate problem-solving skills training and modelling. Programme duration should be determined by clients' mastery in implementing problem-solving skills with their own problems, so that there are opportunities to increase self-efficacy and self-esteem.

\section{ACKNOWLEDGEMENTS}

The authors acknowledge the late Dr Michael J. Kelleher, Founder of the National Suicide Research Foundation, Cork, Ireland. The National Suicide Research Foundation, Ireland was a unit of the Irish Health Research Board.

\section{DECLARATION OF INTEREST}

None.

\section{REFERENCES}

Amsel, L. \& Mann, J. (2001). Suicide risk assessment and the suicidal process approach. In Understanding Suicidal Behaviour: The Suicidal Process Approach to Research, Treatment and Prevention (ed. K. van Heeringen), pp. 163-181. John Wiley: Chichester.

Arensman, E., McAuliffe, C., Corcoran, P. \& Perry, I. (2004). Correspondence. Psychological Medicine 34, 1143-1144.

Arensman, E. \& Kerkhof, A. (1996). Classification of attempted suicide: a review of empirical studies, 1963-1993. Suicide and Life-Threatening Behaviour 26, 46-67.

Beck, A. T., Schuyler, D. \& Herman, I. (1974a). Development of suicidal intent scales. In The Prediction of Suicide (ed. A. T. Beck, H. L. P. Resnik and D. J. Lettieri), pp. 45-56. The Charles Press: Philadelphia.

Beck, A. T., Ward, C. H., Mendelson, M., Mock, J. \& Erbaugh, J. (1961). An inventory for measuring depression. Archives of General Psychiatry 4, 561-571.

Beck, A. T., Weissman, A., Lester, D. \& Trexler, L. (1974b). The measure of pessimism: the hopelessness scale. Journal of Consulting and Clinical Psychology 42, 861-865.

Bernadt, M. W., Mumford, J., Taylor, C., Smith, B. \& Murray, R. M. (1982). Comparison of questionnaire and laboratory tests in the detection of excessive drinking and alcoholism. Lancet 6, 325-328.

Bille-Brahe, U., Kerkhof, A. J. F. M., De Leo, D., Schmidtke, A., Crepet, P., Lönnqvist, J., Michel, K., Salander-Renberg, E., Stiles, T. C., Wasserman, D. \& Egebo, H. (1996). A repetitionprediction study on European parasuicide populations. Crisis 17, 22-31.

Bille-Brahe, U., Schmidtke, A., Kerkhof, A. J. F. M., De Leo, D., Lönnqvist, J. \& Platt, S. (1994). Background and introduction to the study. In Attempted Suicide in Europe: Findings from the Multicentre Study on Parasuicide by the WHO Regional Office for Europe (P.10). (ed. A. J. F. M. Kerkhof, A. Schmidtke, U. BilleBrahe, D. De Leo and J. Lönnqvist), pp. 3-15. DSWO Press: Leiden.

Castells, M. A. \& Furlanetto, L. M. (2005). Validity of the CAGE questionnaire for screening alcohol-dependent inpatients on hospital wards. Revista Brasiliera de Psiquiatria 27, 54-57.

Chan, D. W. (1985). The Chinese version of the General Health Questionnaire: does language make a difference? Psychological Medicine 15, 147-155.

Dieserud, G., Røysamb, E., Braverman, M. T., Dalgard, O. S. \& Ekeberg, Ø. (2003). Predicting repetition of suicide attempt. A prospective study of 50 suicide attempters. Archives of Suicide Research 7, 1-17.

Dori, G. A. \& Overholser, J. C. (1999). Depression, hopelessness, and self-esteem: Accounting for suicidality in adolescent psychiatric inpatients. Suicide and Life Threatening Behaviour 29, 309-318.

Ewing, J. A. (1984). Detecting alcoholism: the CAGE questionnaire. Journal of the American Medical Association 252, 1905-1907.

Fleming, J. S. \& Courtenay, B. E. (1984). The dimensionality of selfesteem. Some results for a college sample. Journal of Personality and Social Psychology 46, 404-421.

Goldberg, D. P., Rickels, K., Downing, R. \& Hesbacher, P. (1976). A comparison of two psychiatric screening tests. British Journal of Psychiatry 129, 61-67 (30-item version as used in EPSIS II). (GHQ-30 obtained through the NFER Nelson Publishing Company, Darville House, 2 Oxford Road East, Windsor, SL4 1DF, Berks.)

Goldberg, D. \& Williams, P. (1988). A User's Guide to the General Health Questionnaire. NFER Nelson: London.

Goodchild, M. E. \& Duncan-Jones, P. (1985). Chronicity and the General Health Questionnaire. British Journal of Psychiatry 146, 55-61.

Hawton, K., Arensman, E., Townsend, E., Bremner, S., Feldman, E., Goldney, B., Gunnell, D., Hazell, P., Van Heeringen, K., House, A., Owens, D., Sakinofsky, I. \& Traskman-Bendz, L. (1998) Deliberate self-harm: systematic review of efficacy of psychosocial treatments in preventing repetition. British Medical Journal 317, 441-447. 
Hawton, K. \& Fagg, J. (1995). Repetition of attempted suicide: the performance of the Edinburgh predictive scales in patients in Oxford. Archives of Suicide Research 1, 261-272.

Hawton, K., Fagg, J., Simkin, S., Bale, E. \& Bond, A. (1997). Trends in deliberate self-harm in Oxford 1985-1995: implications for clinical services and the prevention of suicide. British Journal of Psychiatry 171, 556-560.

Hawton, K., Kingsbury, S., Steinhardt, K., James, A. \& Fagg, J. (1999). Repetition of deliberate self-harm by adolescents: the role of psychological factors. Journal of Adolescence 22, 369-378.

Henriques, G. R., Brown, G. K., Berk, M. S. \& Beck, A. T. (2004). Marked increases in psychopathology found in a 30-year cohort comparison of suicide attempters. Psychological Medicine $\mathbf{3 4}$ 833-841.

Hjelmeland, H. (1996). Repetition of parasuicide: a predictive study. Suicide and Life-Threatening Behaviour 26, 395-404.

Joiner, T. E. \& Rudd, M. D. (2000). Intensity and duration of suicidal crises vary as a function of previous suicide attempts and negative life events. Journal of Consulting and Clinical Psychology 68 909-916.

Kehrer, C. A. \& Linehan, M. M. (1996). Interpersonal and emotional problem solving skills and parasuicide among women with borderline personality disorder. Journal of Personality Disorders 10, 153-163.

Kerkhof, A. J. F. M. \& Arensman, E. (2004). Repetition of attempted suicide: frequent but hard to predict. In Suicidal Behaviour: Theories and Research Findings (ed. D. De Leo, U. Bille-Brahe, A. J. F. M. Kerkhof and A. Schmidtke), pp. 111-124. Hogrefe \& Huber: Gottingen.

Kerkhof, A. J. F. M., van Egmond, M., Bille-Brahe, U., Platt, S. \& Schmidtke, A. (1993). European Parasuicide Study Interview Schedule (EPSIS II). In Facts and Figures (ed. U. Bille-Brahe, T. Bjerke, D. Crepet, D. De Leo, C. Haring, K. Hawton, A. J. F. M. Kerkhof, J. Lönnqvist, K. Michel, A. Phillipe, X. Pommereau, I. Querejeta, E. Salander-Renberg, A. Schmidtke, B. Temesváry, D. Wasserman and J. G. Sampaio Faria). Who/Euro EUR/ICP/PSF 018: Copenhagen.

Keyes, S. (1984). Gender stereotypes and personal adjustment: Employing the PAQ, TSBI and GHQ with samples of British adolescents. British Journal of Social Psychology 23, 173-180.

Lewinsohn, P. M., Rohde, P. \& Seeley, J. R. (1994). Psychosocial risk factors for future adolescent suicide attempts. Journal of Consulting and Clinical Psychology 62, 297-305.

Linehan, M. M., Camper, P., Chiles, J. A., Strosahl, K. \& Shearin, E. L. (1987). Interpersonal problem-solving and parasuicide. Cognitive Therapy and Research 11, 1-12.

McLeavey, B. C., Daly, R. J., Murray, C. M., O'Riordan, J. \& Taylor, M. (1987). Interpersonal problem-solving deficits in self-poisoning patients. Suicide and Life-Threatening Behaviour 17, 33-49.

Milnes, D., Owens, D. \& Blenkiron, P. (2002). Problems reported by self-harm patients: Perception, hopelessness, and suicidal intent. Journal of Psychosomatic Research 53, 819-822.

Owens, D., Dennis, M., Read, S. \& Davis, N. (1994). Outcome of deliberate self-poisoning: An examination of risk factors for repetition. British Journal of Psychiatry 165, 797-801.

Petrie, K., Chamberlain, K. \& Clarke, D. (1988). Psychological predictors of future suicidal behaviour in hospitalized suicide attempters. British Journal of Clinical Psychology 27, 247-257.

Platt, S., Bille-Brahe, U., Kerkhof, A., Schmidtke, A., Bjerke, T., Crepet, P., De Leo, D., Haring, C., Lönnqvist, J., Michel, K., Philippe, A., Pommereau, X., Querejeta, I., Salander-Renberg, E., Temesvary, B., Wasserman, D. \& Sampaio Faria, J. (1992).
Parasuicide in Europe. The WHO/EURO multicentre study on parasuicide I. Introduction and preliminary analysis for 1989. Acta Psychiatrica Scandinavica 85, 97-104.

Pollock, L. R. \& Williams, J. M. G. (1998). Problem solving and suicidal behaviour. Suicide and Life-Threatening Behaviour 28 375-387.

Pollock, L. R. \& Williams, J. M. G. (2004). Problem-solving in suicide attempters. Psychological Medicine 34, 163-167.

Rietdijk, E. A., van den Bosch, L. M., Verheul, R., Koeter, M. W. \& van den Brink, W. (2001). Predicting self-damaging and suicidal behaviors in female borderline patients: Reasons for living, coping, and depressive personality disorder. Journal of Personality Disorders 15, 512-520.

Rosenberg, M. (1965). Society and the Adolescent Self-image. Princeton University Press: Princeton.

Rotheram-Borus, M. J., Trautman, P. D., Dopkins, S. C. \& Shrout, P. E. (1990). Cognitive style and pleasant activities among female adolescent suicide attempters. Journal of Consulting and Clinical Psychology 58, 554-561.

Rudd, M. D., Joiner, T. \& Rajab, M. H. (1996). Relationships among suicide ideators, attempters and multiple attempters in a young adult sample. Journal of Abnormal Psychology 105, 541-550.

Sakinofsky, I. \& Roberts, R.S. (1990). Why parasuicides repeat despite problem resolution. British Journal of Psychiatry 156, 399-405.

Sakinofsky, I., Roberts, R. S., Browne, Y., Cumming, C. \& James, P. (1990). Problem resolution and repetition of parasuicide: a prospective study. British Journal of Psychiatry 156, 395-399.

Sanderman, R. \& Ormel, J. (1992). De Utrechtse Coping Lijst (UCL): validiteit en betrouwbaarheid. Gedrag en Gezondheid 20 , $32-37$.

Sandin, B., Chorot, P., Santed, M., Valiente, R. \& Joiner, T. (1998) Negative life events and adolescent suicidal behaviour: a critical analysis from a stress process perspective. Journal of Adolescence 21, 415-426.

Schreurs, P. J. G., Van de Willige, G., Brosschot, J. F., Tellegen, B. \& Graus, G. M. H. (1993). De Utrechtse Coping Lijst. Herziene Handleiding (revised manual). Swets en Zeitlinger: Lisse.

Schreurs, P. J. G., Van de Willige, G., Tellegen, B. \& Brosschot, J. F. (1988). De Utrechtse Copinglijst: Handleiding (manual). Swets en Zeitlinger: Lisse.

Townsend, E., Hawton, K., Altman, D. G., Arensman, E., Gunnell, D., Hazell, P., House, A. \& Van Heeringen, K. (2001). The efficacy of problem-solving treatments after deliberate self-harm: metaanalysis of randomized controlled trials with respect to depression, hopelessness and improvement in problems. Psychological Medicine 31, 979-988

Tyrer, P., Thompson, S., Schmidt, U., Jones, V., Knapp, M., Davidson, K., Catalan, J., Airlee, J., Baxter, S., Byford, S., Byrne, G., Cameron, S., Caplan, R., Cooper, S., Ferguson, B., Freeman, C., Frost, S., Godley, J., Greenshields, J., Henderson, J., Holden, N., Keech, P., Kim, L., Logan, K., Manley, C., MacLeod, A., Murphy, R., Patience, L., Ramsay, L., De Munroz, S., Scott, J., Seivewright, H., Sivakumar, K., Tata, P., Thornton, S., Ukoumunne, O. C. \& Wessely, S. (2003). Randomized controlled trial of brief cognitive behaviour therapy versus treatment as usual in recurrent deliberate self-harm: the POPMACT Study. Psychological Medicine 33, 969-976.

Williams, M. (1997). Cry of Pain: Understanding Suicide and Selfharm. Penguin: London.

Zahl, D. \& Hawton, K. (2004). Repetition of deliberate self-harm and subsequent suicide risk: Long term follow-up study of 11,583 patients. British Journal of Psychiatry 185, 70-75. 